Revue d'histoire de l'Amérique française

REVUE D.HISTOIRE DE L'AMÉRIQUE FRANÇAISE

\title{
A mari usque ad mare - La grande saga canadienne
}

\section{Serge Courville}

Volume 42, numéro 3, hiver 1989

URI : https://id.erudit.org/iderudit/304709ar

DOI : https://doi.org/10.7202/304709ar

Aller au sommaire du numéro

Éditeur(s)

Institut d'histoire de l'Amérique française

ISSN

0035-2357 (imprimé)

1492-1383 (numérique)

Découvrir la revue

Citer cette note

Courville, S. (1989). A mari usque ad mare - La grande saga canadienne. Revue d'histoire de l'Amérique française, 42(3), 429-439.

https://doi.org/10.7202/304709ar d'utilisation que vous pouvez consulter en ligne.

https://apropos.erudit.org/fr/usagers/politique-dutilisation/ 
NOTE CRITIQUE

\title{
A MARI USQUE AD MARE - LA GRANDE SAGA CANADIENNE ${ }^{1}$
}

\author{
SERGE COURVILLE \\ Département de géographie \\ Université Laval
}

\begin{abstract}
Dans ce miroir qu'est l' histoire, nous voyons par-delà l'étroit présent et discernons la mesure des choses. Sans elle, nous perdons le souffle de notre esprit. Si nous voilons notre histoire, elle vient nous surprendre à notre insu...
\end{abstract}

Karl Jaspers, Initiation à la méthode philosophique

Bien peu de pays se sont dotés d'un atlas historique. Bien peu aussi ont eu les moyens d'assumer de façon aussi éclatante leur bilinguisme. Le Canada comptera désormais parmi ceux-là. Inspiré d'une longue tradition scientifique qui remonte aux pionniers de la géographie historique anglaise et canado-américaine, cet atlas est plus qu'une synthèse des faits et des événements qui ont façonné le Canada: il est un véritable monument élevé à la mémoire du passé, un tribut payé aux découvreurs et aux fondateurs du pays, qu'il s'agisse des premiers Autochtones ou des Européens qui sont venus par la suite s'y établir. Il aura fallu plus de dix ans d'efforts pour concrétiser ce beau rêve. Dix années de recherche, d'échanges, de correspondance, généreusement soutenues financièrement par le Conseil de recherches en sciences humaines du Canada et diverses universités canadiennes, dont principalement celle de Toronto, pour produire ce qui apparaît déjà comme l'un des faits marquants de cette fin de siècle dans le monde de l'édition savante. Ce travail immense, démesuré, aura coûté, quand il sera complété, plusieurs millions de dollars canadiens et rallié plusieurs douzaines de chercheurs venus d'horizons très divers.

\footnotetext{
1 À propos de l'Atlas historique du Canada - Tome I: Des origines à 1800 (Montréal, Presses de l'Université de Montréal, 1987), 95,00\$.
} 
Les premiers résultats sont consignés dans le volume 1 de ce qui deviendra en fait un triptyque. Intitulé «Des origines à 1800», il s'ouvre sur le retrait de la dernière grande nappe glaciaire voilà plus de 10000 ans et se referme plusieurs millénaires plus tard, à l'aube du XIXe siècle de notre ère. C'est ce volume qui sera commenté ici, uniquement sous sa version française. Aboutissement d'une oeuvre préparée de longue date par les enseignements scientifiques de Harold Adams Innis et d'Andrew Hill Clark, deux pionniers de la géographie historique du Canada à qui l'ouvrage est dédié, il est introduit par le directeur général de l'atlas, Wm. G. Dean (Géographie, University of Toronto) et l'archiviste fédéral, Jean-Pierre Wallot, et préfacé par les deux principaux maîtres d'oeuvre du projet, R. C. Harris (Géographie, University of British Columbia), directeur du volume, et G. J. Matthews (University of Toronto), responsable de la cartographie et du graphisme pour l'ensemble de l'atlas. Il résulte de l'effort concerté de plusieurs chercheurs canadiens dont huit réunis en comité de rédaction et responsables chacun de plusieurs planches: Louise Dechêne (Histoire, Université McGill), également directrice de l'édition française, W. J. Eccles (Histoire, University of Toronto), C. E. Heidenreich (Géographie, York University), A. G. Macpherson (Géographie, Memorial University of Newfoundland), D. W. Moodie (Géographie, University of Manitoba), R. I. Ruggles (Géographie, Queen's University), B. G. Trigger (Anthropologie, Université McGill), et J. V. Wright (Commission archéologique du Canada, Musée canadien des civilisations). Quant à la traduction française, elle a été placée sous la responsabilité de Marcel Paré (Université de Montréal). Sont venus également s'ajouter une foule d'autres collaborateurs (conseillers, assistants-étudiants, diplômés, cartographes, dessinateurs, personnel de secrétariat, etc.), trop nombreux pour être cités ici, mais dont ce fut presque l'unique ambition au cours de cette dernière décennie de réussir l'impossible. Car il faut le dire, à la fin des années 1970, la connaissance du passé canadien n'était pas celle d'aujourd'hui. En outre, comme le premier volume de l'atlas devait composer avec deux univers radicalement différents, l'Amérique indigène et celle des Européens et, dans ce dernier cas, avec deux espaces-temps issus de deux cultures différentes, le défi était de taille, beaucoup plus grand peut-être que dans toute autre synthèse d'histoire canadienne.

\section{UNE OEUVRE IMMENSE, À FONDEMENT IDENTITAIRE}

Cette première publication donne déjà un avant-goût de ce que sera le projet de l'Atlas historique du Canada une fois complété. Une oeuvre immense, impressionnante, de très grande qualité scientifique, qui se pose déjà en oeuvre obligée de référence en histoire canadienne. Cette oeuvre sera d'autant plus prisée que, par-delà le rêve qu'elle concrétise, celui des chercheurs qui y ont cru, elle donne une assise 
nouvelle à celui d'un pays constitué politiquement voilà un peu plus d'un siècle mais dont on a pu douter par moments qu'il puisse jamais exister tant était grande la distance qui séparait ses frontières et ses univers humains constitutifs. Une oeuvre majeure donc, une oeuvre de prestige aussi, et peut-être même à cause de cela, une oeuvre éminemment politique, un «projet», destiné à atténuer les différences entre les cultures et à fournir les fondements identitaires à l'expression d'un nationalisme canadien. Cela n'enlève rien à sa qualité d'oeuvre savante, ni même à sa légitimité comme entreprise scientifique. Toutefois, cela colore et éclaire son contenu, d'autant plus que son message est extrêmement puissant, fondé non seulement sur une intelligence des faits qui ont conduit à l'élaboration de ce grand empire nordique qu'est devenu le Canada, mais également sur une présentation très soignée de ces faits qui donne la pleine mesure du talent scientifique canadien. Aussi ce premier volume plaira-t-il au grand public canadien, en plus de projeter une image positive du Canada à l'étranger.

Dès le départ, l'atlas a voulu stimuler la recherche, devenir une sorte de catalyseur pour les différentes équipes de chercheurs oeuvrant à travers le pays. Il a bien joué son rôle, suscitant maintes enquêtes, maints bilans qui auraient sûrement mis plus de temps à être réalisés. Leur but ultime? Nourrir une cartographie que l'on voulait la plus attrayante et la plus innovatrice possible, où l'écrit allait se conjuguer à l'image pour offrir une vision dynamique du passé. Le résultat final est impressionnant: plusieurs dizaines de planches toutes plus riches les unes que les autres et une collection non moins impressionnante de textes introductifs et explicatifs qui en présentent la séquence et en résument le contenu. Et surtout, un savoir bien construit, capable de stimuler la conscience canadienne, car l'ouvrage ne s'adresse pas qu'à l'intelligence, il interpelle aussi les sentiments.

On sait la puissance évocatrice de l'image dans nos sociétés actuelles. L'on sait aussi le sentiment de fierté que suscite chez tout individu ou chez tout groupe humain le fait de connaître ses origines. Parce qu'il donne une profondeur historique à l'expérience canadienne et une allure très «moderne» au passé, l'atlas apparaît comme un moyen puissant de nourrir la mémoire collective et de susciter un tel sentiment, un peu comme les oeuvres mobilisatrices d'autrefois qui voyaient dans la connaissance et l'attachement au passé le moyen de créer un citoyen nouveau, conscient de ses racines et capable, de ce fait, d'affronter l'avenir. «L'Histoire peut nourrir un peuple», disait le chanoine Groulx. Mais il est vrai que son époque en était une de grands bouleversements; bien des choses ont changé depuis. La nôtre, pourtant, n'est pas exempte de difficultés: ne songeons qu'aux défis qu'ont posés (et que continuent de poser) à l'unité canadienne les tensions politiques des vingt ou trente dernières années, ou à ceux que posent (et que continueront de poser) à la «culture» canadienne celle de nos voisins du sud. L'atlas est une 
réponse à ces menaces, inconsciente peut-être, mais une réponse tout de même, qui pose implicitement qu'en dépit de ses découpages administratifs et culturels le pays est «un», historiquement «distinct» de tout ce qui l'entoure et dont l'avenir repose sur un idéal «commun». Certes, ce ne sont pas là des énoncés clairement exprimés, mais on en sent toute la présence, bien soutenus par un appareil didactique aussi riche qu'original.

\section{UN PUISSANT OUTIL DIDACTIQUE}

C'est l'une des grandes qualités de cet atlas d'être très beau visuellement. Conçu comme un livre aussi bien à consulter qu'à feuilleter, à lire qu'à regarder, il plaira tant aux jeunes qu'aux moins jeunes, aux initiés qu'aux profanes, qui ne trouveront pas toujours là une matière facilement accessible, mais qui seront fascinés par la qualité générale de l'ouvrage et par la quantité impressionnante d'informations colligées. Aux jeunes, il fournira l'occasion d'un premier contact avec l'histoire canadienne; aux moins jeunes, celle d'une mise à jour de faits ou d'événements déjà appris mais depuis longtemps oubliés; aux profanes, celle d'un apprentissage nouveau basé sur le progrès des connaissances; aux initiés, celle d'une réflexion critique qui les amènera peut-être à de nouvelles recherches ou à un renouvellement de perspective. Mais c'est sans doute auprès des enseignants du niveau pré-universitaire que cet atlas aura le plus de succès, comme matériel de base pour les cours d'Histoire du Canada ou comme ouvrage d'appoint à ceux déjà disponibles ou sur le point de l'être. Car il faut dire qu'il y a peu d'outils de ce genre dans les écoles et les collèges, les livres de géographie ne comportant pas beaucoup de contenu historique et les livres d'histoire étant rarement aussi bien illustrés. Ce sera l'un des mérites de cet ouvrage de combler cette lacune, en présentant quantité de sujets pour lesquels la matière de base sera réunie et en offrant quantité de moyens de les présenter.

Une autre grande qualité de ce volume est d'être très riche sur le plan du contenu. Au total, il comprend 69 planches regroupées sous six grandes en-têtes introduites chacune par un texte général de présentation. À l'exception de la première et de la dernière parties, où la couverture territoriale est plus large, partout ailleurs la démarche est régionale, fondée sur les découpages administratifs actuels ou sur les regroupements suggérés par la géographie. La première partie est consacrée à la préhistoire et aux Autochtones et présente de façon très détaillée la lente pénétration et l'occupation du territoire par les chasseurs venus d'Asie, avec plusieurs planches consacrées à leurs premiers établissements, ceux qui suivront, leurs pratiques, leurs systèmes d'échanges, leur culture; elle inclut également une planche consacrée aux voyages et aux établissements des Norois. La deuxième partie est consacrée à la région atlantique et s'ouvre avec les premières grandes explorations 
européennes et leurs premiers établissements de pêche; elle se referme sur la déportation des Acadiens et sur les paysages de la fin du XVIIIe siècle. La troisième nous fait pénétrer vers l'intérieur du continent, avec divers développements allant des premières missions européennes jusqu'au commerce des fourrures et aux rivalités impériales; elle s'achève sur la Guerre de Sept ans, la guerre avec les Amérindiens et l'invasion américaine de la fin du XVIIIe siècle. La quatrième est consacrée à la colonie laurentienne qu'elle présente en quelques touches consacrées à la population, à l'économie et à l'habitat, ainsi qu'aux missions amérindiennes de la vallée du Saint-Laurent. La cinquième est consacrée au Nord-Ouest et au commerce des fourrures, avec plusieurs vues originales sur les grandes zones économiques, les postes de traite, les explorations, le transport et la population autochtone. Quant à la sixième partie, qui ne comprend que deux planches, elle donne une image de l'est du Canada vers 1800 et une synthèse des peuplements autochtones vers 1820 .

Chaque partie, chaque planche est un monde en soi, une sorte de fresque commentée des faits et des événements qui se sont déroulés sur le territoire canadien ou dans ses débordements immédiats. Et la perspective étant spatiale, tout devient alors très concret, enrichi de dessins, de graphiques et de gravures anciennes ou récentes, qui donnent un air tantôt d'époque, tantôt très actuel à l'ensemble. Aucun effort n'a été ménagé pour rendre ces planches attrayantes, et même lorsqu'elles paraissent trop chargées pour être aisément accessibles au lecteur, elles restent fascinantes. Aussi l'effet de représentation est-il extrêmement puissant, beaucoup plus que ne le sera jamais un livre, si bien construit et si bien présenté soit-il.

L'ouvrage pourtant n'est pas sans limites, d'autant moins que sa perspective est très large, la quantité d'informations présentées très élevée et les clientèles visées très nombreuses. De plus, comme il comporte une dimension identitaire, c'est autant d'aspects qu'il faut maintenant aborder.

\section{TOUT DE MÊME, DES DIFFICULTÉS}

En effet, quiconque s'attarde au contenu détaillé du volume ne peut manquer de constater les difficultés que les auteurs ont affrontées. La préface en résume quelques-unes, en rappelant que si un atlas peut parfois avancer en éclaireur, il ne peut le plus souvent que suivre et réfléter l'état de la recherche; il dépend de la documentation disponible. Par ailleurs, comme l'histoire canadienne malgré tout n'est pas simple, il fallait faire des choix, d'autant plus difficiles que les écoles de pensée au Canada sont nombreuses et pas toujours facilement conciliables. Enfin, il fallait tout à la fois dresser un bilan satisfaisant de la recherche et en vulgariser convenablement les résultats, cela au moment même 
où s'annonçaient de nouveaux paradigmes en histoire et de nouvelles thématiques de recherche, inspirant des travaux dont les fruits ne seraient manifestement pas disponibles avant la parution de l'atlas. C'est donc avec de réelles contraintes qu'il a fallu composer, les principales demeurant peut-être les difficultés d'intégration de l'ensemble, l'état de la recherche vers la fin des années 1970 et la difficulté de tenir compte des évolutions enregistrées depuis. C'est qu'en raison de l'ampleur du projet et des échéances strictes auxquelles ils étaient soumis, les concepteurs ont dû très tôt arrêter leurs choix, ce qui les a privés de cette souplesse pourtant si nécessaire aux grandes oeuvres.

Il ne saurait être question de commenter ici le contenu et la présentation matérielle de chaque planche, laissons cela aux spécialistes, il y en aura sûrement. Laissons aussi aux sondeurs d'opinion le soin d'en apprécier l'accueil chez les enseignants ou le grand public. Attardons-nous plutôt à l'orientation générale de l'ouvrage et à ce qui le caractérise, et d'abord à ses objectifs. On peut en compter au moins trois qui ont dominé tous les autres. Le premier est implicite: il s'agissait de charger de profondeur humaine un territoire que l'histoire a mis plusieurs siècles à élaborer sur le plan politique. Les deux autres sont plus explicites, à savoir: redonner toute leur place aux Autochtones et élargir les perspectives du Canada colonial, ceci, en privilégiant les circonstances économiques et sociales de la vie quotidienne plutôt que les événements géopolitiques et leurs conséquences territoriales.

Dans l'ensemble, ces objectifs ont été atteints: le Canada apparaît comme un pays qui a bel et bien un passé et que l'espace et les événements historiques unissent «d'une mer à l'autre»; en outre, pour la première fois peut-être dans un tel ouvrage savant, les Autochtones retrouvent sinon toute leur place, du moins une place plus qu'honorable, beaucoup plus grande en fait que dans aucune autre synthèse historique, compte tenu de l'état de la recherche archéologique et de la difficulté pour les Blancs de pénétrer l'univers amérindien; enfin, on a assez bien réussi à montrer la place du Canada colonial dans l'économie-monde nord-atlantique. Toutefois, on n'a pas vraiment réussi une présentation intégrée de l'histoire canadienne, pas plus que l'on a réussi à dégager le vrai visage de ceux qui nous ont précédés. Car à l'exception de quelques planches ou parties de planches qui montrent les interactions entre les différents facteurs qui composent la trame de notre histoire, ou de celles orientées plus franchement vers la vie de tous les jours et les influences qui la dominent, la plupart, sinon l'immense majorité d'entre elles, s'intéressent plutôt aux macro-phénomènes et aux superstructures de la vie collective, en plus de les présenter sur des bases que seule la chronologie des événements permet parfois de raccorder.

En effet, ce qui frappe le plus dans cet ouvrage, c'est l'écart qui sépare le type de traitement réservé à la préhistoire et aux Autochtones 
de celui réservé au Canada colonial et plus particulièrement à la société qui l'a vu naître. C'est aussi le type de découpage géographique adopté pour la présentation des faits, dont la segmentation trahit la réalité de certains régimes juridiques et politiques de l'époque. Enfin, c'est l'importance accordée à la «Grande» Histoire dans la présentation du Canada colonial et, à l'intérieur de cette «Grande» Histoire, le peu de place réservée aux faits ou aux événements qui échappent à la logique du rapport colonies-métropoles, mais qui peuvent rendre compte également de bien des choses. Tous ces choix laissent dans l'ombre bien des développements, à commencer par ceux qui ont contribué à la mise en place du Canada initial ou qui ont nourri la vie des communautés de base. Surtout, ils projettent une image du Canada qui convient bien sans doute à un objectif didactique (un pays construit d'est en ouest et imbriqué dans un réseau d'interrelations avec l'Europe), mais qui donne finalement une image assez limitée de l'expérience «canadienne» et de sa patiente adaptation au temps, aux événements et à l'espace.

Entendons-nous bien, il ne s'agit pas ici de nier l'intérêt d'une démarche qui a voulu s'ouvrir à l'ensemble du territoire canadien. Il ne s'agit pas non plus de nier l'intérêt d'une approche qui a voulu montrer la contribution de chacune des régions au façonnement du Canada, ni celui d'une recherche orientée vers les faits qui ont dominé la vie collective. Il s'agit plutôt de faire remarquer que ce qui est central dans l'histoire canadienne des XVIIe et XVIIIle siècles n'apparaît pas ici dans toute son ampleur, pas plus d'ailleurs que les véritables circonstances de la vie quotidienne. En outre, en optant pour une présentation régionale de ces faits plutôt que pour celle de leurs aires juridiques ou économiques initiales d'expression, l'atlas met de l'avant des contributions qui n'appartiennent pas vraiment aux ensembles ou sous-ensembles où ceux-ci sont illustrés. Enfin, en s'intéressant surtout aux événements qui ont dominé la vie collective, il passe sous silence bien des aspects pourtant tout aussi marquants dans l'expérience humaine des premiers Canadiens.

L'une des plus belles réussites de ce volume réside sans contredit dans la place faite aux Autochtones où, en dépit d'un exposé parfois très spécialisé, trop pour un public non initié, l'on sent un réel effort de synthèse, beaucoup plus grand peut-être que partout ailleurs dans l'ouvrage. Abordé avec sympathie, le sujet est introduit chaque fois que le contexte ou les circonstances l'exigent, un peu comme un tribut à payer aux premiers vrais «fondateurs» du pays. C'est la partie forte de l'atlas, avec le traitement réservé à la pêche et au commerce des fourrures, où l'ancienneté de la recherche, associée à la qualité et à la quantité de chercheurs qui ont oeuvré sur le sujet, ont permis de stimulantes présentations; et peut-être aussi avec celui réservé à la région de l'Atlantique, où l'on sent bien également cette volonté d'assumer le poids des événements historiques. Mais c'est aussi une partie chargée de sens, où 
la reconnaissance d'une culture pourtant bien différente et bien antérieure dans ses origines à l'idée même d'un «Canada» vient, pour ainsi dire, donner l'un de ses premiers fondements à une identité. En soi, cette reconnaissance est justifiée: les Autochtones n'ont-ils pas été les premiers occupants du territoire, ou du moins de ce qui deviendra plus tard le territoire canadien? Toutefois, elle prend une teinte particulière quand on constate le traitement réservé à la société laurentienne, la première en fait, avec la société acadienne, à devenir «canadienne» dans les faits et à donner son nom et ses premiers traits au Canada d'origine européenne. C'est non seulement le risque d'une vision mythique des origines (nos «ancêtres» les Amérindiens) qui est en cause ici, favorisée par l'ampleur même de la présentation réservée aux Indigènes (près du tiers des planches, sans compter tous les autres cartons insérés à l'intérieur de celles-ci), mais celui d'une vision voilée de l'histoire où l'on ne discerne plus la mesure des choses.

Nul ne niera l'effort déployé par l'atlas pour rappeler la présence et le rôle de la France dans ce qui constitue aujourd'hui le territoire canadien. Nul ne niera non plus l'intérêt des thématiques abordées. Pourtant, qui demain saisira la place réelle et le rôle de la NouvelleFrance, de ce Canada du Régime français en fait, dans la définition du Canada actuel? C'est toute une réalité politique, juridique, économique, sociale, culturelle qui s'estompe ici, sous l'effet d'une cartographie éclatée qui ne nous en fait voir que des parcelles reconstituées autour d'entités juridiques encore inexistantes à l'époque, mais dont on finit par croire qu'elles eurent un rôle important à jouer dans la mise en place du Canada initial. C'est tout le contraire du traitement réservé à l'Ontario, par exemple, qui, jusqu'à la fin du XVIIIe siècle, n'est à l'origine d'aucun développement, mais à qui l'atlas réserve pourtant une place importante qui décompose et déstructure en fait celle de la Nouvelle-France, comme si l'empire français d'Amérique ne s'était limité qu'aux rives du Saint-Laurent. Car il ne faut pas prendre en compte ici que l'écrit, encore que les mots puissent aussi être porteurs de sens voir notamment la planche 3 qui présente les vestiges des premiers groupes paléoindiens sous l'intitulé «L'Ontario méridional, 8600 av. J.-C.»; on ne retrouve aucune autre mention semblable pour le Québec dans les titres, sauf dans la planche consacrée aux batailles de Québec de 1759-1760. Il faut aussi tenir compte de la puissance évocatrice d'une image qui multiplie les cartes consacrées à la région des Grands-Lacs sans toujours les recontextualiser par rapport à cet ensemble plus vaste qu'était la Nouvelle-France. Bien sûr, l'on compte bien quelques cartons qui en tracent le contour et il a bien fallu composer avec la géographie (la position des Grands-Lacs dans le territoire ontarien). Il n'en demeure pas moins qu'un sentiment de vide persiste, qui pèsera lourd demain dans les représentations que l'on se fera du Canada initial. On peut même se demander dans ce contexte quel support l'atlas 
pourra offrir à l'enseignement du fait français au Canada? C'est qu'en plus de restreindre la place de cette Nouvelle-France, il met l'accent sur un type d'histoire qui laisse dans l'ombre bien des aspects, en plus de renforcer indirectement certains mythes, par exemple celui du «coureur des bois», rendu d'autant plus crédible ici que le traitement réservé au commerce des fourrures est important.

On sait le rôle joué par les grands événements dans la mise en place des empires ou des nations. L'on sait aussi l'importance des institutions ou des grandes organisations de commerce ou d'échanges dans l'explication des sociétés. Pourtant, ce qui est signifiant dans le vécu d'un peuple ne relève pas toujours de ce genre d'événements ou d'organisations, et même s'ils éclairent le contexte plus général dans lequel ce vécu s'insère, leur place ne saurait être exclusive, sans quoi l'histoire «nationale» risque fort de se transformer en histoire «coloniale», c'està-dire en celle des nations qui ont exploité le territoire ou qui s'en sont disputé les ressources. C'est le danger qui guette toutes les grandes synthèses historiques, où les conflits, les guerres, les institutions, le grand commerce prennent souvent le pas dans l'explication (et dans la représentation que l'on finit par se faire) de l'histoire et du vécu d'une population, de ses attitudes, de ses valeurs, de son genre de vie..., bref de ce qu'il convient d'appeler sa «culture». L'atlas n'a pas vraiment su éviter ce genre de piège, en dépit d'efforts louables déployés en ce sens. Tout cela restreint la présentation faite de la société coloniale et plus particulièrement de la société laurentienne qui en fut la principale composante.

Des ambitions de la France et de ses entreprises en terre canadienne, on dit beaucoup: grandes explorations, premiers établissements côtiers, puis, de l'intérieur, implantation de cadres institutionnels, poussées évangélisatrices, développement de la pêche et du commerce des fourrures, affrontements avec l'Amérindien et l'Anglais et, finalement, défaite aux mains des armées britanniques après l'épisode douloureux de la déportation acadienne. Par contre, de la société coloniale comme telle, on dit peu: moins en fait que ce que nous ont déjà appris nos livres d'histoire. La difficulté la plus sérieuse à cet égard concerne la société laurentienne. En 1800, cette société forme encore le principal segment de la population canadienne: près de 220000 habitants (dont 190000 francophones) sur les quelque 350000 que comptent alors les sept colonies de l'Amérique du Nord britannique, soit la plus grande part de l'ensemble. Or, ce qu'on en révèle ne tient qu'en une douzaine de planches dont plusieurs consacrées à des thématiques qui n'ont que peu de rapport avec le vécu de cette société: origine géographique de la population initiale, distribution du peuplement en 1739, missions amérindiennes, grand commerce maritime, profils urbains, découpages seigneuriaux, vues de la campagne et de l'agriculture, pêche et architecture domestique. Ce n'est pas que ces thèmes soient inintéressants en 
soi ou que leur présentation cartographique soit déficiente, bien au contraire: plusieurs planches sont même innovatrices et proposent plus d'un élément de solution à des questions demeurées jusqu'ici sans réponse. Mais on se serait attendu à plus et surtout à quelque chose de bien différent, compte tenu de la place et du rôle de cette société dans l'ensemble canadien, plus proche peut-être de la perspective offerte dans l'introduction de la section, nettement plus riche en contenu que la partie cartographique et surtout plus sensible à une présentation intégrée des faits.

Les sujets pourtant ne manquaient pas: modes d'expansion du peuplement et de formation des communautés de base, organisation de l'écoumène, stratégies d'appropriation et de contrôle du sol, processus de reproduction sociale, définition de réseaux locaux d'échanges, rapports avec les colonies voisines, tensions diverses reliées à la Conquête et, plus tard, à l'apparition de nouveaux peuplements culturellement et socialement différents, compétitions qui en découlent, émergence, dans la seconde moitié du XVIIIe siècle, d'une nouvelle socio-économie qui porte en elle les germes de transformations futures, développement d'un savoir-faire qui jouera un grand rôle plus tard dans l'économie, etc., bref tous ces faits que l'on passe généralement sous silence parce qu'on les connaît mal, mais qui expliquent eux aussi le Canada.

Ce qui a plutôt manqué ici, outre peut-être une plus grande participation des chercheurs québécois, c'est une fenêtre ouverte sur la territorialité du groupe humain concerné. Pour cela, il aurait fallu changer d'échelle, pénétrer de l'intérieur le réseau de relation existant au sein de cette société et entre elle et le monde extérieur, pour en mesurer la cohésion et en apprécier l'apport dans l'histoire et la culture canadiennes. Non seulement dans le contexte particulier des XVIIe et XVIIIe siècles, mais en regard également du XIXe siècle qui s'annonce. C'est là un type de recherche que l'atlas n'a pas su susciter ou n'a pas pu ou voulu intégrer, faute de temps ou de moyens, ou tout simplement parce que les travaux en cours semblaient trop peu avancés ou trop éloignés des préoccupations premières des concepteurs. Quelle qu'en ait été la cause, l'effet reste le même, celui d'une société «vue de haut» où, à quelques rares exceptions près, le paysage imposé domine le paysage investi et où les formes et les fonctions de l'espace organisé prennent le pas sur la vie de relation qui reste celle finalement que la métropole orchestre. C'est peu pour illustrer tout ce que cette société a apporté à la culture et à l'identité canadienne! Et ce qui vaut ici pour la société laurentienne vaut également pour les autres sociétés dont la présentation n'est pas très différente, somme toute, de la précédente.

C'est l'une des principales limites de l'ouvrage, l'autre étant cette vision éclatée de la Nouvelle-France qui en décompose la réalité dans l'espace. Elles rendent bien mineures les difficultés soulevées par le 
manque d'arrière-plan contextuel de certaines planches dont on aurait aimé qu'elles précisent les réseaux de migrations ou d'échanges situés à l'amont des faits représentés, de manière à mieux définir les traits de la population d'origine et à mieux caractériser sa vie de relation. Elles rendent plus mineures encore les quelques coquilles relevées ici et là au fil des pages et qui ont échappé à l'attention des responsables: tout au plus quelques fautes de frappe ou de traduction (celle d' «historical géographer» notamment), auxquelles vient s'ajouter une malencontreuse séparation de couleur qui transforme en victoire britannique la dernière victoire française de la Guerre de Sept ans, celle du 28 avril 1760 à Sainte-Foy...

Cela dit, tout limité qu'il soit dans ses perspectives et tout classique qu'il puisse paraître dans certains de ses contenus, le bilan laissé par ce premier volume reste impressionnant. La quantité d'informations colligées, la qualité générale de leur présentation, le caractère innovateur de certaines planches et le nombre de contributions obtenues en demeurent les qualités principales. En outre, il convie à la réflexion et au dépassement, en des termes qu'il n'était peut-être pas encore possible d'imaginer à l'époque où fut conçu le projet, mais qui deviendront importants dans les cycles de recherche à venir. Enfin, même si cette oeuvre bilingue n'atténue pas les différences entre les cultures, elle montre tout l'intérêt du travail en équipe: lui seul finalement pourra faire converger les perspectives scientifiques. Pouvait-on souhaiter plus belle contribution au progrès des connaissances et de la recherche au Canada? 